\title{
Momentum spectra of charmonium produced in a quark-gluon plasma
}

\author{
R. L. Thews \\ Department of Physics, University of Arizona, Tucson, Arizona 85721, USA \\ M. L. Mangano \\ PH Department, TH Unit, CERN, CH-1211 Geneva 23, Switzerland \\ (Received 30 May 2005; published 24 January 2006)
}

\begin{abstract}
We calculate rapidity and transverse momentum distributions of charmonium formed in high-energy heavy ion collisions from incoherent recombination of charm quarks. The results are very sensitive to the corresponding distributions of the charm quarks, and thus can serve as a probe of the state of matter produced in the heavy ion collision. At one extreme, we generate charm pair momenta directly from pQCD amplitudes, which are appropriate if one can neglect the interaction of the quarks with the medium. At the other extreme, we generate momenta of charm quarks in thermal equilibrium with the expanding medium, appropriate for an extremely strong interaction. Explicit predictions are made for $J / \psi$ formation in Au-Au interactions at the BNL Relativistic Heavy Ion Collider. We find that for the case in that charm quark momenta are unchanged from the pQCD production calculation, both the rapidity and transverse momentum spectra of the formed $J / \psi$ are substantially narrower than would be anticipated in scenarios that do not include the in-medium formation. In particular, the average transverse momentum of the $J / \psi$ will exhibit a nonmonotonic behavior in the progression from $p p$ to $p A$ to $A A$ interactions.
\end{abstract}

DOI: 10.1103/PhysRevC.73.014904

PACS number(s): 12.38.Mh

\section{INTRODUCTION}

The behavior of heavy quarkonium states as an experimental signature of medium modification in high-energy heavy ion collisions originated with the prediction of $J / \psi$ suppression [1], which follows from screening of the colorconfinement potential above the phase transition temperature. The suppression argument requires that the probability for recombination of the $c$ and $\bar{c}$ quark is negligible. Recently it has been realized that for energies available at the BNL Relational Heavy Ion Collider (RHIC) and the CERN Large Hadron Collider (LHC), there can be a modification of the suppression argument $[2,3]$.

Consider a central heavy ion collision in which multiple $c \bar{c}$ pairs are produced in binary nucleon-nucleon interactions. If these quarks are then immersed in a medium (such as a quarkgluon plasma) in which a charm quark from one initial $c \bar{c}$ pair can readily interact with an anticharm quark from a different initial $c \bar{c}$ pair, one expects that the number of $J / \psi$ formed from such interactions will be proportional to the total number of $c \bar{c}$ combinations. The relative efficiency of this quadratic process must be normalized by the number of interactions involving charm plus light-flavor quarks in which open-charm hadrons are formed. The normalization measure is generally taken [4] to be proportional to the total number of charged hadrons produced in the heavy ion collision $N_{\mathrm{ch}}$, which leads to $N_{J / \psi} \propto N_{c \bar{c}}{ }^{2} / N_{\mathrm{ch}}$. (Of course, this behavior must saturate before $N_{J / \psi}$ becomes comparable in magnitude to $N_{c \bar{c}}$ ). Then at sufficiently large $N_{c \bar{c}}$, this quadratic behavior must dominate over the color-screening suppression.

Initial estimates [5] from extrapolations of the low-energy charm production measurements and predictions of pQCD indicated of $N_{c \bar{c}} \approx 10$ for central RHIC collisions and several hundred at LHC. Measurements at RHIC of high transverse momentum leptons in Au-Au collisions by PHENIX [6] imply that $N_{c \bar{c}} \approx 20$, and measurement of reconstructed $D$ mesons in $d$-Au collisions by STAR [7] require $N_{c \bar{c}} \approx 40$. Statistical and systematic uncertainties are large enough such that these two measurements are consistent, but the general trend indicates a more rapid growth with energy than initially estimated [8].

Two distinct realizations of this mechanism for heavy quarkonium formation have been developed. In the statistical hadronization model, quarks are distributed into hadrons during the hadronization transition according to chemical equilibrium ratios. The total number of heavy quarks is assumed to be determined by initial production, and an extra fugacity factor $\gamma_{f}$ is determined by overall flavor conservation. In the case of hidden flavor such as $J / \psi$, two powers of $\gamma_{c}$ modify the thermal equilibrium density, which leads to the expected quadratic dependence on $N_{c \bar{c}}[9,10]$. There is no explicit prediction for the $J / \psi$ momentum spectrum, but it is natural to assume a thermal distribution in this model at the deconfinement temperature $T_{c} \approx 170 \mathrm{MeV}$. It is noteworthy that ratios involving radially excited states such as $\frac{\psi^{\prime}}{J / \psi}$ retain their unmodified statistical values.

The kinetic formation model [3,4,11-13] assumes that heavy quarkonium formation takes place during the entire lifetime of a color-deconfined phase. Predictions for the resulting population require the specification of cross sections for formation and dissociation in the medium. The motivation for such a scenario in the case of $J / \psi$ formation has received support from recent lattice calculations of spectral functions. These indicate that $J / \psi$ and $\eta_{c}$ will exist in a thermal environment at temperatures well above the deconfinement transition $[14,15]$. Note that this property is likely valid only for the most deeply bound states. Thus, unlike the statistical model, one does not expect a simple relation to exist between the $J / \psi$ and $\psi^{\prime}$ populations. Also in contrast with statistical 
hadronization, the momentum spectrum of formed $J / \psi$ will reflect the initially produced charm quark spectra plus any modification due to interaction with medium.

In principle, both of these formation processes could occur sequentially. Considerable progress has been made [16-18] in such a scenario when both bound and unbound charm populations evolve in a thermal fireball.

In the next section, we review the basic properties of kinetic in-medium formation of heavy quarkonium, using a specific model for $J / \psi$ which exhibits the expected general properties. We previously found [19] that with the initial PHENIX data from RHIC, we can constrain the model parameters within a fairly broad range, but we can not confirm or rule out this type of formation. A more detailed test must await comparison with the momentum spectra of the formed $J / \psi$. Section III contains details of the two specific charm quark distributions which we consider. One is calculated directly from the initial production process using Next-to-Leading Order (NLO) pQCD amplitudes to generate a set of $c \bar{c}$ pair events appropriate for RHIC energy. The other generates a corresponding set of events which would be expected if the charm quarks subsequently come into local thermal equilibrium with the expanding medium before forming the $J / \psi$. In Sec. IV we present a detailed model calculation of the normalized rapidity and transverse momentum spectra for in-medium $J / \psi$ formation, using charm quark spectra calculated from the pQCD amplitudes. It is important to keep in mind that we are searching for a signature in the $J / \psi$ rapidity and transverse momentum spectra which would indicate the presence of in-medium formation and which is independent of the absolute magnitude of the formation process. Our results are shown to be quite robust with respect to substantial variations in the model parameters. Section V A contains a specific for RHIC results, where we use data for $p p$ and $d$-Au interactions to constrain some of the model parameters. Section V B presents the corresponding calculations using charm quarks in local thermal equilibria. Comparisons with predictions of other models and a summary of results complete this presentation.

\section{FORMATION OF $J / \psi$ IN A COLOR-DECONFINED MEDIUM}

We first examine the net number of $J / \psi$ produced in a color-deconfined medium due to the competing reactions of (a) formation involving recombination of $c$ and $\bar{c}$ and (b) dissociation of $J / \psi$ induced by interactions with constituents of the medium. The simplest dissociation reaction utilizes absorption of single deconfined gluons in the medium to ionize the color singlet $J / \psi, g+J / \psi \rightarrow c+\bar{c}$, resulting in a $c \bar{c}$ pair in a color octet state. This process was originally proposed [20] as a dynamic counterpart of the static color screening effect. The inverse of this process then serves as the corresponding formation reaction, in which a $c \bar{c}$ pair in a color octet state emits a color octet gluon and falls into the color singlet $J / \psi$ bound state.

One can then follow the time evolution of charm quark and charmonium numbers according to a Boltzmann equation in which the formation and dissociation reactions compete, that is,

$$
\frac{d N_{J / \psi}}{d t}=\lambda_{\mathrm{F}} N_{c} N_{\bar{c}}[V(t)]^{-1}-\lambda_{D} N_{J / \psi} \rho_{g},
$$

with $\rho_{g}$ the number density of gluons in the medium. The reactivity $\lambda$ is the product of the reaction cross section and initial relative velocity $\left\langle\sigma v_{\text {rel }}\right\rangle$ averaged over the momentum distribution of the initial participants, i.e. $c$ and $\bar{c}$ for $\lambda_{F}$ and $J / \psi$ and $g$ for $\lambda_{D}$. The gluon density is determined by the equilibrium value in the medium at each temperature, and the volume must be modeled according to the expansion and cooling profiles of the heavy ion interaction region.

This equation has an analytic solution in the case where the total number of formed $J / \psi$ is much smaller than the initial number of $N_{c \bar{c}}$.

$$
N_{J / \psi}\left(t_{f}\right)=\epsilon\left(t_{f}\right)\left[N_{J / \psi}\left(t_{0}\right)+N_{c \bar{c}}^{2} \int_{t_{0}}^{t_{f}} \lambda_{\mathrm{F}}[V(t) \epsilon(t)]^{-1} d t\right],
$$

where $t_{0}$ and $t_{f}$ define the lifetime of the deconfined region. Note that the function $\epsilon\left(t_{f}\right)=\exp \left[-\int_{t_{0}}^{t_{f}} \lambda_{D} \rho_{g} d t\right]$ would be the suppression factor in this scenario if the formation mechanism were neglected.

The initial calculations [4] used the ratio of nucleon participants to participant density calculated in a Glauber model to define a transverse area enclosed by the boundary of the region of color deconfinement. This is supplemented by longitudinal expansion starting at an initial time $t_{0}=0.5 \mathrm{fm}$. Transverse expansion was initially neglected but has been included in subsequent calculations [19]. The expansion was taken to be isentropic, which determines the time evolution behavior of the temperature $T(t)$. The initial value $T_{0}$ is taken as a parameter, and the final $T_{f}$ is fixed at the hadronization point.

The reactivities $\lambda_{F}$ and $\lambda_{D}$ require specification of cross sections. For $\sigma_{D}$ we use the one-pion-exchange Operator Product Expansion (OPE)-based model of gluon dissociation of deeply bound heavy quarkonium [21-23], which is related via detailed balance to the corresponding $\sigma_{F}$. Written in terms of the heavy quarkonium reduced mass $\mu\left(=m_{Q} / 2\right)$, gluon momentum in the $c \bar{c}$ rest frame $k$, and binding energy $\epsilon_{0}$, the dissociation cross section is

$$
\sigma_{D}=\frac{2 \pi}{3}\left(\frac{32}{3}\right)^{2}\left(\frac{2 \mu}{\epsilon_{0}}\right)^{1 / 2} \frac{1}{4 \mu^{2}} \frac{\left(k / \epsilon_{0}-1\right)^{3 / 2}}{\left(k / \epsilon_{0}\right)^{5}} .
$$

This expression assumes that the heavy quarkonium has a nonrelativistic Coulomb bound state spectrum with $\epsilon_{0} \gg$ $\Lambda_{\mathrm{QCD}}$, and utilizes an operator product expansion in the large $N_{c}$ limit. These cross sections for $J / \psi$ kinematics are shown in Fig. 1. One sees that they are peaked at low energy, and that $\sigma_{F}>\sigma_{D}$ because of the large binding energy (we used the vacuum value $\epsilon_{0} \approx 600 \mathrm{MeV}$ ).

We should note that the approximations concerning a nonrelativistic Coulomb bound state spectrum are somewhat marginal for the charmonium case, although they are better justified for the $v$ states. Therefore, for applications to $J / \psi$ we will investigate the effects of a considerable range of 


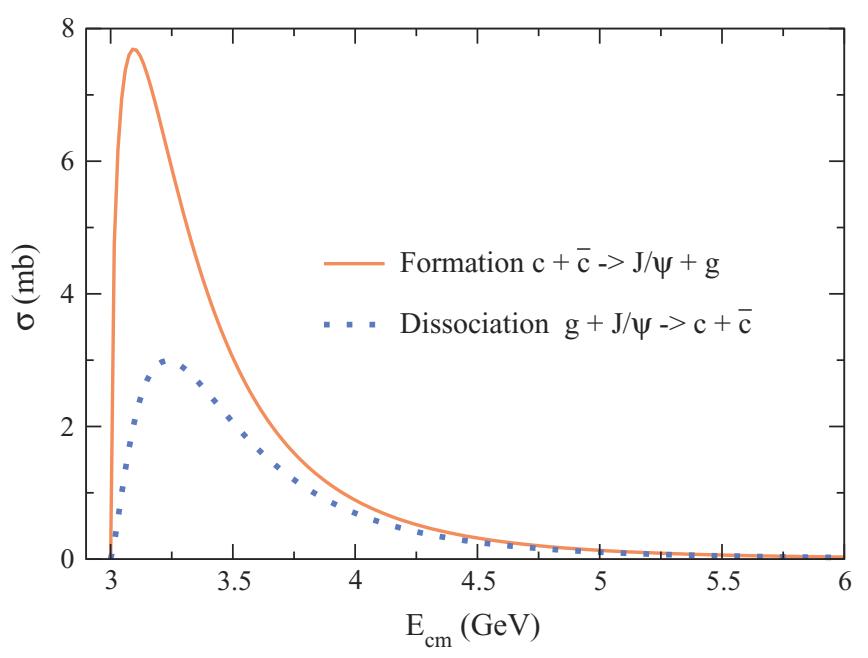

FIG. 1. (Color online) OPE-based cross sections for $J / \psi$ formation and dissociation.

alternative cross-section behavior, as has been done in a number of treatments of $J / \psi$ production in a purely hadronic scenario [24-27].

The initial model calculations for $J / \psi$ at RHIC [4] yielded interesting predictions for the centrality behavior of the final population $N_{J / \psi}\left(t_{f}\right)$ normalized by $N_{c \bar{c}}$, or equivalently normalized by the number of binary collisions. Because of the quadratic behavior of the formation rates with $N_{c \bar{c}}$, one expects an increase with centrality of formed $J / \psi$ per binary collision, if the formation reaction dominates. The primary variation in these predictions was due to the initial charm quark momentum distributions, which enter through the calculation of the formation and dissociation reactivities as defined in Eq. (1). Differences of up to a factor of 5 or more resulted as the charm quark momentum distributions varied from thermal (produced the largest formation efficiency) through perturbative QCD distributions. There may be additional uncertainties in overall magnitude. In particular, an implementation of this process in a transport model calculation [28] did not result in a large effect at RHIC energy.

The first confrontation with experiment involved the initial PHENIX measurements of $J / \psi$ at $200 \mathrm{GeV}$ [29]. Values of $d N_{J / \psi} / d y(y=0)$ for three centrality bins were extracted, but only the most central bin value yielded an upper bound because of the limited of statistics. Although these limitations prevented a definitive test of the formation model, it was clear that the predictions which followed from thermal charm quark distributions were disfavored. The pQCD charm quark distributions led to formation rates which were roughly consistent with experiment. A parameter scan involving initial temperature, initial charm quark production, transverse expansion velocity of the deconfined region, and initial $J / \psi$ production number found a surprisingly large region of parameter space within which the formation mechanism results were consistent with the PHENIX data points and limits.

It is interesting that these data are also consistent with binary-scaled $J / \psi$ production in $p p$ interactions [30], modified with a small suppression factor. Given the sensitivity of the formation process to initial charm production (both magnitude and momentum spectra), even the improved statistics anticipated for the PHENIX Au-Au data currently undergoing analysis may not be adequate to differentiate between these distinct possibilities. Fortunately, the momentum spectra for the produced $J / \psi$ will also be measured. In anticipation of this information, we then proceed to extend the calculations of the kinetic formation process to include the rapidity and transverse momentum spectra of the final $J / \psi$ population.

We return to the expression for in-medium formation of $J / \psi$ in Eq. (1), modified to calculate the momentum spectra of the formed $J / \psi$. Rather than specifying a functional form for the charm quark momentum distributions, we will generate a sample of $c \bar{c}$ events appropriate to a given physical situation and then sum over the sample to calculate the differential formation reactivity:

$$
\frac{d N_{J / \psi}}{d^{3} P_{J / \psi}}=\int \frac{d t}{V(t)} \sum_{i=1}^{N_{c}} \sum_{j=1}^{N_{\bar{c}}} \mathrm{v}_{\mathrm{rel}} \frac{d \sigma}{d^{3} P_{J / \psi}}\left(P_{c}+P_{\bar{c}} \rightarrow P_{J / \psi}+X\right) .
$$

Note that the formation magnitude exhibits the explicit quadratic dependence on $N_{c \bar{c}}$ via the double summation.

The formation rate (differential in $J / \psi$ momentum) is integrated over time to get the spectra of in-medium formed $J / \psi$. We assume for simplicity that (a) the spatial density of charm and anticharm quarks are equal within the deconfinement region, and (b) the charm quark momentum spectra are independent of time. The time-independence assumption could in principle be relaxed, at the expense of a more involved calculational scheme. For now, we simply present the results as limiting cases which bracket a range of possibilities between the two types of charm momentum distributions considered. Then the differential $J / \psi$ spectra just involve an integral of the inverse deconfinement volume over time, which is irrelevant for the normalized momentum spectra. Of course, these spectra will be modified by the dissociation process [second term in Eq. (1)], as well as the spectra of $J / \psi$ which are initially produced. We consider the effects of the dissociation process in Sec. IV.

The differential dependence of the formation cross section which follows from a detailed balance of the dissociation cross section in Eq. (3) is extracted from the analog of atomic photoionization. In this case, the relevant amplitude involves the coupling of a gluon with the $J / \psi$ color electric dipole of the quarkonium, again in the approximation of a Coulombic bound state wave function. The procedure to evaluate the $J / \psi$ momentum spectra from Eq. (4) just involves a scan for each $c \bar{c}$ over final state phase space, weighted by the appropriate differential cross section. To determine the sensitivity of the resulting $J / \psi$ spectra to this particular differential cross section, we also performed the calculations using an isotropic invariant amplitude, normalized to the same OPE-based total cross section. We find in general that the effects on the $J / \psi$ momentum spectra are negligible. Comparison of results will be shown in Sec. IV. 


\section{CHARM QUARK MOMENTUM SPECTRA}

\section{A. Perturbative QCD calculations}

The perturbative QCD spectra are obtained using the results of the full $\mathcal{O}\left(\alpha_{s}^{3}\right)$ calculation, as implemented in the event generator described in [31]. In the case of nuclear beams, the parton distribution functions of the proton have been modified to account for nuclear effects, using the model of Ref. [32]. The terms of $\mathcal{O}\left(\alpha_{s}^{3}\right)$ corresponding to real-emission diagrams will generate a nontrivial transverse momentum distribution for the $c \bar{c}$ pair. We generated samples of unweighted events, which will be used in the following analyses. To ensure that all events in the samples have positive weight, we merged the negative-weight events arising from the cancellation of soft and collinear singularities [31] with the sample of positive weight events having a transverse momentum of the $c \bar{c}$ pair $p_{T}(c \bar{c})<0.4 \mathrm{GeV}$, and we smeared their $p_{T}(c \bar{c})$ over the $0-0.4 \mathrm{GeV}$ range. $c \bar{c}$ pairs selected from the same event will be labeled as "diagonal" pairs: They dominate the $J / \psi$ formation in $p p$ and $p A$ collisions, where production of multiple pairs is negligible. In the case of $A A$ collisions, multiple hard scatterings can give rise to several $c \bar{c}$ pairs, and charm (anticharm) quarks from independent pairs are allowed to form a $J / \psi$. These uncorrelated pairs are randomly selected from our sample of unweighted events, reproducing on average the correct kinematic distributions of such pairs.

Shown in Fig. 2 are the shapes of the transverse momentum distributions. ${ }^{1}$ We plot the spectra of individual charm quarks (with and without inclusion of nuclear effects in the PDFs), as well as the transverse momenta of $c \bar{c}$ pairs. Here and in the following the generic term " $c \bar{c}$ pairs" refers to summing over all possible pairings of $c$ and $\bar{c}$ quarks in an event, in the limit

${ }^{1} \mathrm{~A}$ recent review of the overall theoretical systematics at $\mathcal{O}\left(\alpha_{s}^{3}\right)$ of the charm spectrum in the RHIC energy range is given in Ref. [33].

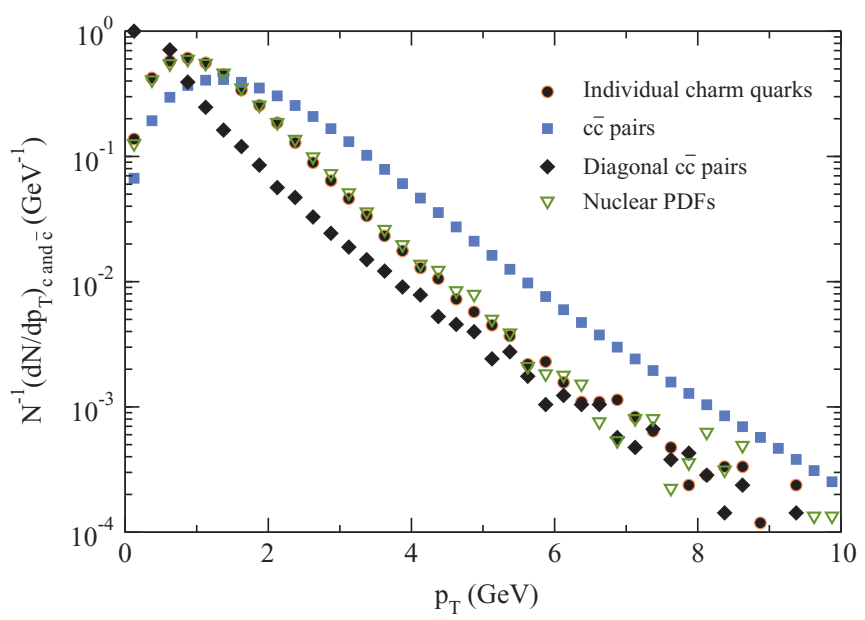

FIG. 2. (Color online) Transverse momentum distributions from the NLO pQCD $c \bar{c}$ events at RHIC200 energy. Since we use a sample of some $80 \mathrm{~K} c \bar{c}$ events, the distribution of all pairs is dominated by the off-diagonal pairs. Also shown is the individual charm quark distribution generated using parton distribution functions (PDFs) modified for nuclear effects by the EKS98 [32] factors.

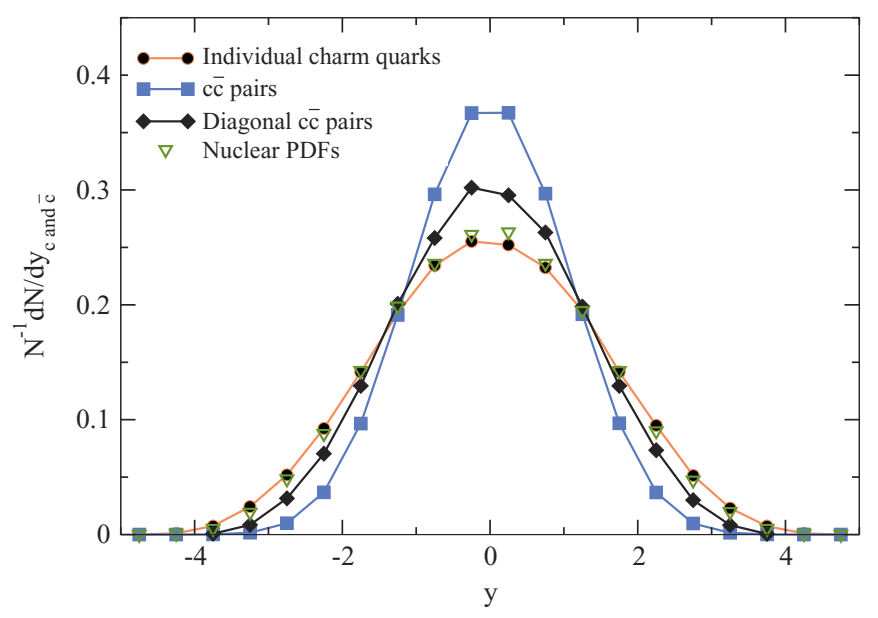

FIG. 3. (Color online) Same as Fig. 2, but for rapidity distributions from the NLO pQCD $c \bar{c}$ events at RHIC200 energy.

of large $N_{c \bar{c}}$ multiplicity. The term "diagonal pairs" will refer instead to $c \bar{c}$ pairs coming from the same hard collision.

The nuclear effects are essentially negligible in the individual charm quark distributions (compare the solid circles and open triangles - the scatter at large $p_{T}$ reflects statistical fluctuations due to our finite sample size). The $c \bar{c}$ pair distribution is approximately twice as wide as that for individual quarks (as measured by $\left\langle p_{T}^{2}\right\rangle$ ). This is a result of the independence of the transverse momentum vectors (both in size and in azimuthal direction) for off-diagonal pairs of $c$ and $\bar{c}$ quarks produced in different hard scatterings. For diagonal pairs, the relative azimuthal angle is $\pi$ for all LO events. The small nonzero $\left\langle p_{T}^{2}\right\rangle$ for diagonal pairs thus arises entirely from NLO effects. The mean values $\left\langle p_{T}{ }^{2}\right\rangle$ of these distributions are $2.45 \mathrm{GeV}^{2}$ for the individual quarks, $4.88 \mathrm{GeV}^{2}$ for all pairs, and $1.20 \mathrm{GeV}^{2}$ for the diagonal pairs.

Shown in Fig. 3 are the corresponding rapidity distributions. Again the nuclear effects are negligible in the individual charm quark distributions. The $c \bar{c}$ pair distributions are both narrower than that for individual quarks. The diagonal pair distribution is somewhat wider that that of all pairs, which again follows from the kinematics of diagonal pairs which have $p_{t}$ (pair) $\approx 0$ from the LO events. These widths can be quantified by the $\Delta y \equiv \sqrt{\left\langle y^{2}\right\rangle-\langle y\rangle^{2}}$ values, 1.46 for individual charm quarks, 1.25 for diagonal pairs, and 1.03 for all pairs.

\section{B. Intrinsic $k_{T}$ effects}

We must now modify the charm quark transverse momentum spectra to include effects of partonic confinement. This could be accomplished by introducing partonic transverse momentum dependence directly into the pQCD calculation. For simplicity, we choose to modify the NLO charm quark results by adding an additional transverse momentum "kick" $k_{T}$ to each quark in a $c \bar{c}$ pair [34]. The magnitude is determined by a Gaussian distribution with width parameter $\left\langle k_{T}{ }^{2}\right\rangle$ and a uniform azimuthal distribution. We will determine the width parameter directly from data. For nuclear collisions, the additional effects of initial state interactions of the nucleons will be included in this parametrization. 


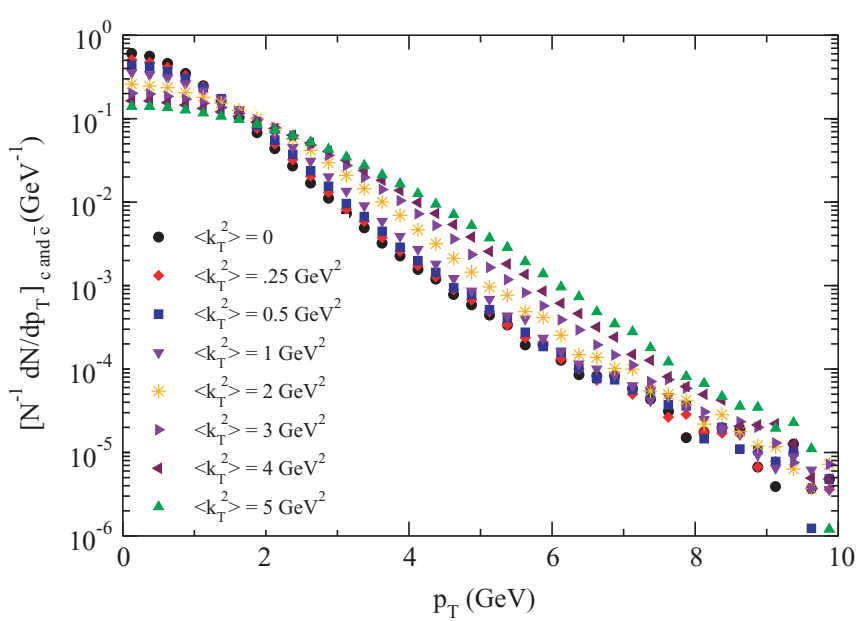

FIG. 4. (Color online) Charm quark transverse momentum distributions including intrinsic $k_{T}$.

The resulting charm quark transverse momentum spectra are shown in Fig. 4 for a range of $\left\langle k_{T}{ }^{2}\right\rangle$. The width of these spectra satisfy the simple pattern

$$
\left\langle p_{T}{ }^{2}\right\rangle\left(\left\langle k_{T}{ }^{2}\right\rangle\right)=\left\langle p_{T}{ }^{2}\right\rangle\left(\left\langle k_{T}{ }^{2}\right\rangle=0\right)+\left\langle k_{T}{ }^{2}\right\rangle .
$$

This pattern is again a result of the uncorrelated relative azimuthal angles between the transverse momentum kick and the transverse momentum of the original pQCD-generated quarks.

Figure 5 shows the rapidity distributions of the charm quark events after the $\left\langle k_{T}{ }^{2}\right\rangle$ modification. Aside from small corrections near midrapidity, these spectra are effectively independent of $\left\langle k_{T}{ }^{2}\right\rangle$, because the charm quark longitudinal momenta are unchanged by addition of the transverse kick.

We can then investigate the systematic behavior of $c \bar{c}$ pair distributions as a function of $\left\langle k_{T}{ }^{2}\right\rangle$. Figure 6 shows the transverse momentum distributions of all pairs and also diagonal pairs. The symbol legends are the same as those in Figs. 4 and 5. The distributions for all pairs (dominated by the off-diagonal pairs) shown by the corresponding solid

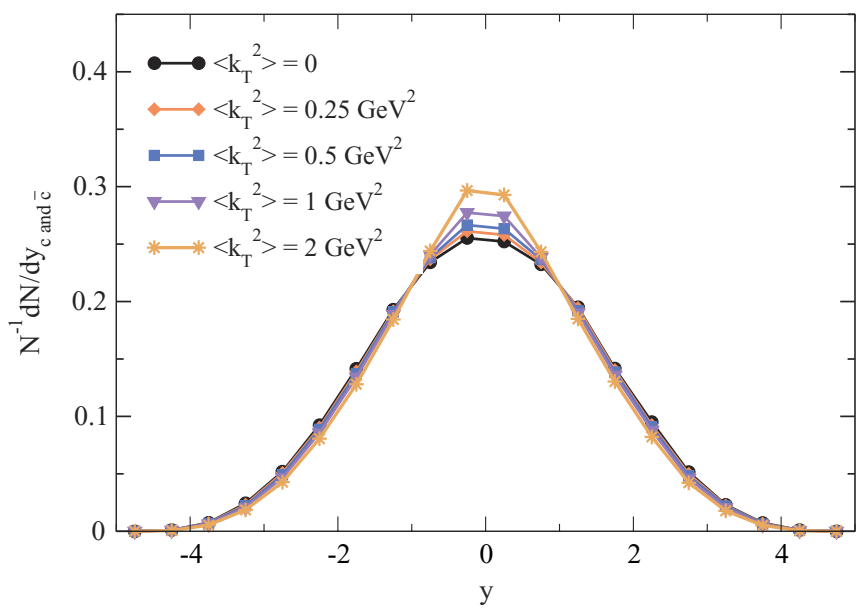

FIG. 5. (Color online) Charm quark rapidity distributions including intrinsic $k_{T}$.

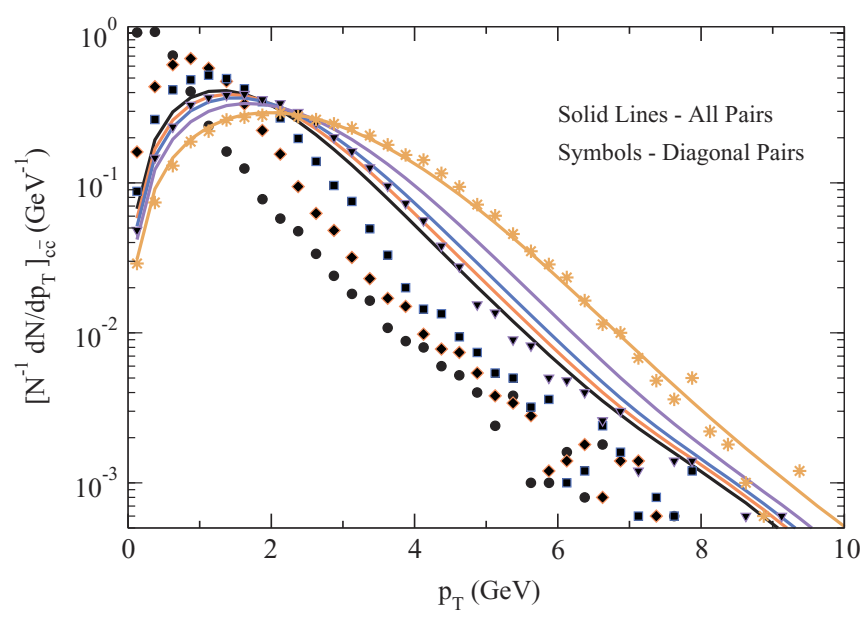

FIG. 6. (Color online) Same as Fig. 5, but for $c \bar{c}$ pair transverse momentum distributions including intrinsic $k_{T}$.

curves are significantly broader than those for the diagonal pairs at $\left\langle k_{T}{ }^{2}\right\rangle=0$, because the pair $p_{T}=0$ for the LO events. However, that difference decreases as $\left\langle k_{T}{ }^{2}\right\rangle$ increases, because the quarks in the off-diagonal pairs receive uncorrelated $k_{T}$ kicks. This is verified by a linear fit to the $\left\langle p_{T}{ }^{2}\right\rangle$ of the pair distributions. For the full range of generated diagonal pairs, one obtains

$$
\left\langle p_{T}{ }^{2}\right\rangle_{\text {diagonal } c \bar{c}}=1.2 \mathrm{GeV}^{2}+4\left\langle k_{T}{ }^{2}\right\rangle,
$$

to be compared with

$$
\left\langle p_{T}{ }^{2}\right\rangle_{\text {all }} c \bar{c}=4.9 \mathrm{GeV}^{2}+2\left\langle k_{T}{ }^{2}\right\rangle
$$

for the sample with all pairs.

It is also evident that the diagonal pair sample exhibits some scatter at the larger $p_{T}$ values on account of statistical fluctuations limited by the total number of generated events, which is of order $10^{4}$. This limitation does not appear until much higher $p_{T}$ for the all pairs sample with the total number of order $10^{8}$. The effect is evident if we truncate the high- $p_{T}$ events in the diagonal pair sample. The resulting width satisfies

$$
\left\langle p_{T}{ }^{2}\right\rangle_{\text {diagonal } c \bar{c}}\left(p_{T}<5 \mathrm{GeV}\right) \approx 0.8 \mathrm{GeV}^{2}+4\left\langle k_{T}{ }^{2}\right\rangle .
$$

This parametrization will be useful in comparison with the initial PHENIX data in $p p$ and $d$-Au interactions, where the measured $p_{T}$ of $J / \psi$ are limited to the $p_{T}$ range in Eq. (8).

The rapidity spectra of the $c \bar{c}$ pairs are shown in Fig. 7, again using the same symbol legends to indicate $\left\langle k_{T}{ }^{2}\right\rangle$. One sees that there is not a dramatic effect in the range of $\left\langle k_{T}{ }^{2}\right\rangle$ up to $2.0 \mathrm{GeV}^{2}$. As $\left\langle k_{T}{ }^{2}\right\rangle$ increases, the width $\Delta y$ decreases by less than $10 \%$ for both the all pairs spectra and the diagonal pairs.

\section{Thermal plus flow charm quark distributions}

We also generated a set of charm quark momenta to investigate the opposite scenario, in which the interaction with the partonic constituents in a deconfined region is sufficiently strong to result in their thermalization in the expanding medium. This scenario is not favored by conventional arguments based on small heavy quark cross sections and the 


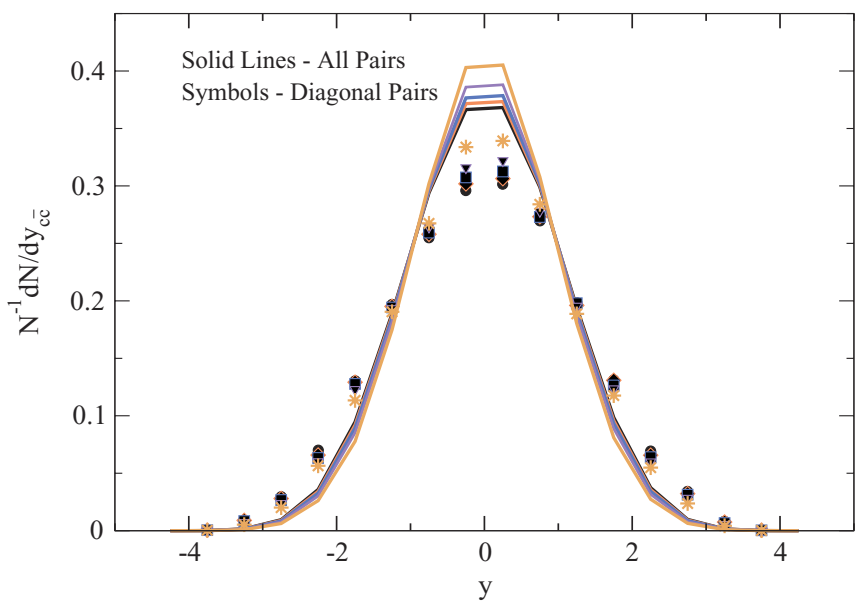

FIG. 7. (Color online) Same as Fig. 5, but for $c \bar{c}$ pair rapidity distributions including intrinsic $k_{T}$.

inhibition of medium-induced radiation due to the dead-cone effect [35]. Analysis of high- $p_{T}$ leptons in Au-Au collisions as a measurement of charm quark production shows they are consistent with spectra unmodified from the initial pQCD calculations [36]. However, it was subsequently shown [37] that the resulting spectra of heavy quark mesons and electrons for $p_{T}<3 \mathrm{GeV}$ cannot distinguish between the two different scenarios. Recently the possibility of heavy quark thermalization was found to be accelerated by interactions involving resonant hadronic states in a plasma [38]. In any event, it is interesting to consider such a heavy quark momentum spectrum in the kinetic formation process to illustrate the impact of this very different scenario on the resulting bound state spectra.

To generate this set of charm quark momenta, we use the distribution which follows from numerical results of transverse-boosted Bjorken boost invariant hydrodynamics [39],

$$
\frac{d N}{d p_{T}^{2}} \propto m_{T} \int_{0}^{R} r d r I_{0}\left(\frac{p_{T} \sinh y_{T}(r)}{T}\right) K_{1}\left(\frac{m_{T} \cosh y_{T}(r)}{T}\right),
$$

where the transverse mass $m_{T} \equiv \sqrt{m^{2}+p_{T}^{2}}$ and $R$ is the transverse system size. The hadronization temperature $T$ and the rapidity of transverse expansion $y_{T}(r)$ are determined from a fit to the light hadrons. Reference [37] determined $T=128 \mathrm{MeV}$ and $y_{T}(r)=\tanh ^{-1}\left(\beta_{T}(r)\right)$ with a linear boost profile for the transverse expansion velocity, $\beta_{T}(r)=\beta_{\max }\left(\frac{r}{R}\right)$ with $\beta_{\max }=0.65$ for central collisions. This form has also been used to fit the transverse momentum spectrum of hadrons in a statistical model in which the freezeout and hadronization temperatures are identical [40]. In this case, the linear profile of the transverse expansion was taken to vary with transverse rapidity rather than velocity, but for typical $\beta_{\max }$ values the difference is not significant. For the calculations here, we use the linear profile in transverse rapidity. We also consider an alternate set of parameters, $T=170 \mathrm{MeV}$ and $\beta_{\max }=0.5$, which emerges from a coalescence study of thermal partons which also fit the light hadron spectra at RHIC [41].

Figure 8 shows the transverse momentum spectra of charm quarks which result from Eq. (9) using the parameter sets

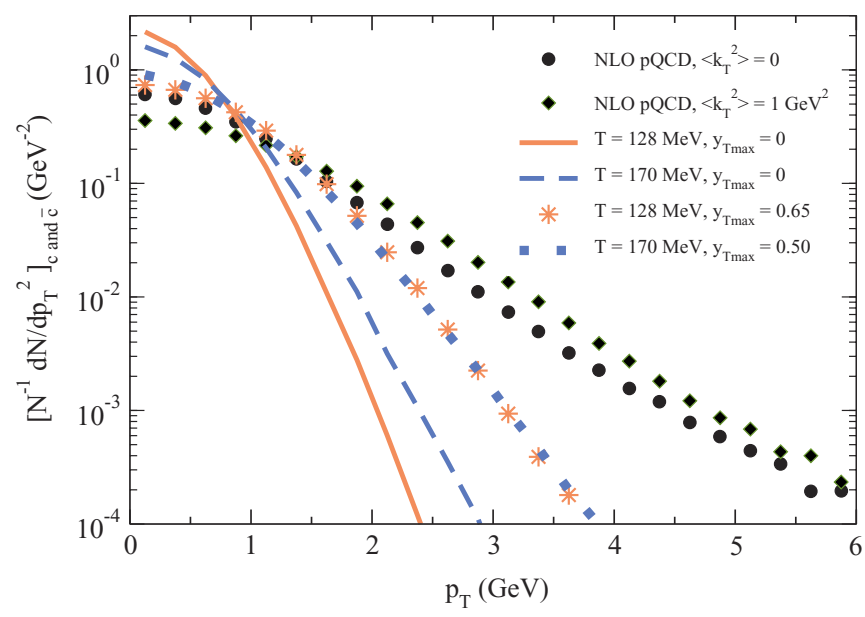

FIG. 8. (Color online) Thermal plus flow charm quark transverse momentum distributions. Shown are thermal without flow (solid and dashed lines), thermal with flow (star and dotted line), and pQCD (symbols).

$\left(T, y_{T_{\max }}\right)=(128 \mathrm{MeV}, 0),(170,0),(128,0.65)$ and $(170,0.50)$. For no flow, the distributions for different temperatures are of course different. However, when augmented by the corresponding transverse flow, the resulting distributions are almost identical, since both $y_{T \max }$ values have been adjusted to fit the observed flow at the corresponding freezeout temperatures.

The pQCD distributions shown for comparison are much broader and have an obvious nonthermal behavior for large $p_{T}$. The widths can be characterized by the calculated $\left\langle p_{T}^{2}\right\rangle$ values of $0.47,0.67,1.35$, and $1.24 \mathrm{GeV}^{2}$ for the thermal and thermal plus flow distributions, compared with 2.45 and $3.45 \mathrm{GeV}^{2}$ for the pQCD examples.

The transverse momentum spectra of the thermal plus flow $c \bar{c}$ pairs are shown in Fig. 9. In this case, there is no set of diagonal pairs since all identity has been lost in the thermalization process. The pair distribution widths are twice those of the corresponding distributions for single charm quarks, as expected because of the uncorrelated relative azimuthal angles. Their $\left\langle p_{T}{ }^{2}\right\rangle$ values are $0.93,1.32,2.70$, and

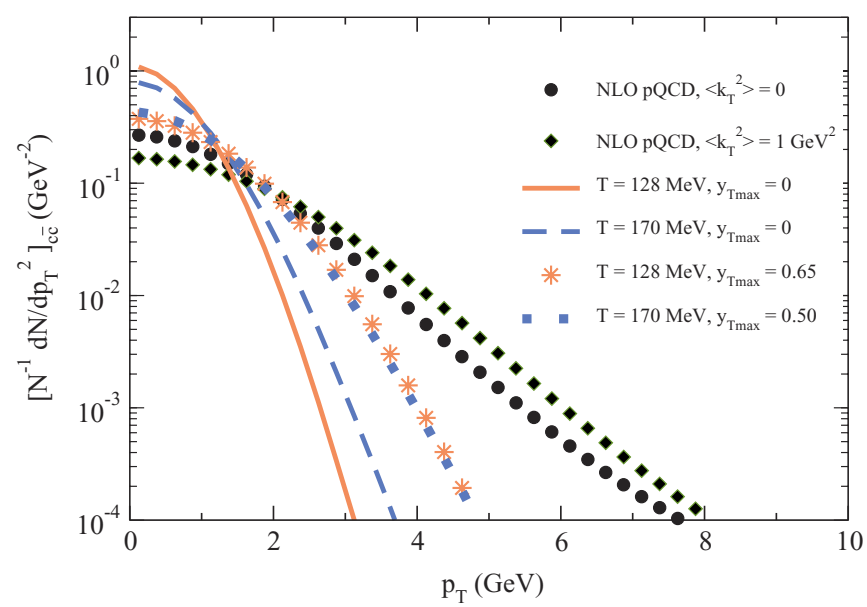

FIG. 9. (Color online) Same as Fig. 8, but for thermal plus flow pair transverse momentum distributions. 
$2.46 \mathrm{GeV}^{2}$, respectively. Again these are much smaller than the pQCD comparison distributions, which have $\left\langle p_{T}{ }^{2}\right\rangle$ of 4.9 and $6.9 \mathrm{GeV}^{2}$.

\section{DETAILS OF KINETIC FORMATION MODEL CALCULATIONS}

We generate the transverse momentum and rapidity spectra of the formed $J / \psi$ according to Eq. (4). For each $c \bar{c}$ pair, we transform it to the pair c.m. system and weight the formation event by $\mathrm{v}_{\text {rel }} \sigma_{F}$. The formation cross section $\sigma_{F}$ is obtained via detailed balance from the dissociation cross section $\sigma_{D}$,

$$
\sigma_{F}=\frac{48}{36} \sigma_{D} \frac{\left(s-M_{J / \psi}{ }^{2}\right)^{2}}{s\left(s-4 m_{c}^{2}\right)},
$$

in terms of the charm quark mass $m_{c}$ and the energy invariant $s$. $\sigma_{D}$ is taken from Eq. (3), with modified threshold gluon momentum to account for the finite mass of the $J / \psi$.

The angular distribution of the $J / \psi$ in the $c \bar{c}$ rest frame is taken from the corresponding behavior of atomic or nuclear [42] photodissociation, which gives

$$
\frac{d \sigma_{F}}{d^{3} p} \propto \sin ^{2} \theta
$$

The sensitivity to the high-energy behavior of $\sigma_{F}$ is probed by imposing a maximum energy cutoff parameter $d_{\max },{ }^{2}$ which limits the invariant mass of the $c \bar{c}$ pairs which can initiate the formation of a $J / \psi$ :

$$
\sqrt{s_{c \bar{c}}}<M_{J / \psi}+d_{\max } .
$$

The resulting rapidity and transverse momentum spectra are given by the black circles compared in Figs. 10 and 11, respectively. Our choice of initial default parameters sets the binding energy $\epsilon_{0}=0.63 \mathrm{GeV}$, the mass cutoff parameter $d_{\max }=3.0 \mathrm{GeV}$, and no $k_{T}$ kick $\left(\left\langle k_{T}{ }^{2}\right\rangle=0\right)$. The curves refer to a sample of $10^{4}$ independent $c \bar{c}$ pairs, resulting in $10^{8}$ possible pairings.

The impact on these distributions of the various dynamic assumptions is shown by three additional curves:

(i) $J / \psi$, isotropic matrix element: The angular dependence in Eq. (11) is taken to be flat.

(ii) Weighted $c \bar{c}$ pairs: We plot the kinematic variables of the $c \bar{c}$ pairs weighted by the $J / \psi$ formation probability $\mathrm{v}_{\mathrm{rel}} \sigma_{F}$, which differs from the formation process by elimination of exact kinematics relating a $c \bar{c}$ pair to the spectrum of $J / \psi$ which follow from the in-medium formation reaction.

(iii) $c \bar{c}$ pairs: We plot the kinematic variables of the $c \bar{c}$ pairs resulting from all combinations of charm quarks as produced in the $\mathrm{PQCD}$ processes.

One sees that for both spectra the $J / \psi$ formation is not sensitive to the angular dependence of the amplitude, as

\footnotetext{
${ }^{2}$ This is equivalent to the cutoff imposed by the color evaporation model at the open-flavor threshold.
}

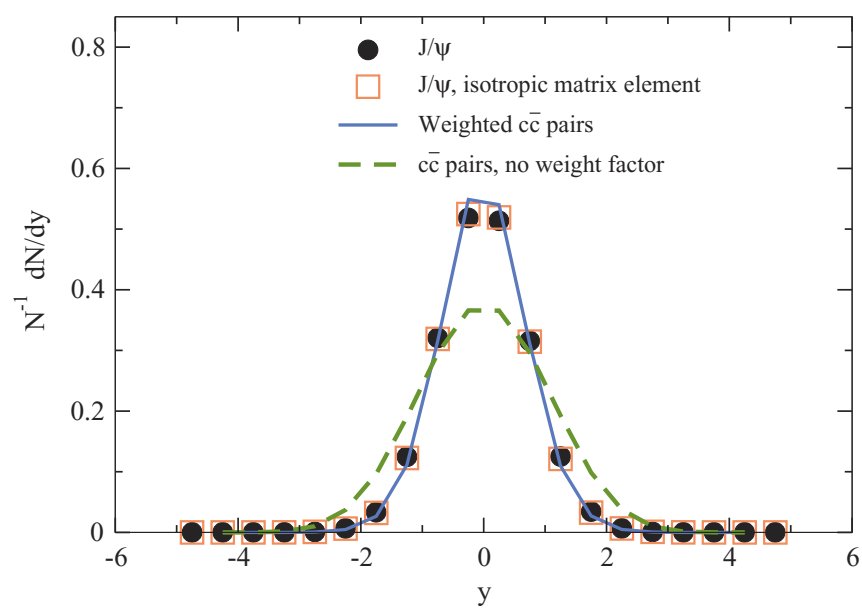

FIG. 10. (Color online) Comparison of rapidity spectra for $c \bar{c}$ pairs and formed $J / \psi$, all using default parameters.

evidenced by the equality of the circle and square plots (except a small effect at the largest values of transverse momentum). The primary effect of the formation dynamics is to produce a narrowing of all spectra, compared with that of the $c \bar{c}$ pairs (dashed line). For the rapidity spectra, even the weighted $c \bar{c}$ pairs (solid line) are identical to the $J / \psi$.

The effects of intrinsic $\left\langle k_{T}{ }^{2}\right\rangle$ on the $J / \psi$ spectra are shown in Figs. 12 and 13. The rapidity spectra are essentially independent of $\left\langle k_{T}{ }^{2}\right\rangle$. The transverse momentum spectra increase with $\left\langle k_{T}{ }^{2}\right\rangle$, as expected. We can parametrize this effect in terms of $\left\langle p_{T}{ }^{2}\right\rangle$ for the formed $J / \psi$ as

$$
\left\langle p_{T}^{2}\right\rangle_{J / \psi}=2.4 \mathrm{GeV}^{2}+\left\langle k_{T}{ }^{2}\right\rangle .
$$

It is interesting to compare this form with the corresponding behavior of all $c \bar{c}$ pairs in Eq. (7), in which the value at $\left\langle k_{T}{ }^{2}\right\rangle=0$ is larger $\left(4.9 \mathrm{GeV}^{2}\right)$ as is the rate of increase (2). A fit of an intermediate case, using all $c \bar{c}$ pairs weighted with the formation cross section $\mathrm{v}_{\mathrm{rel}} \sigma_{F}$, yields an intermediate result,

$$
\left\langle p_{T}{ }^{2}\right\rangle_{c \bar{c} \text { (weighted) }}=3.2 \mathrm{GeV}^{2}+1.5\left\langle k_{T}{ }^{2}\right\rangle \text {. }
$$

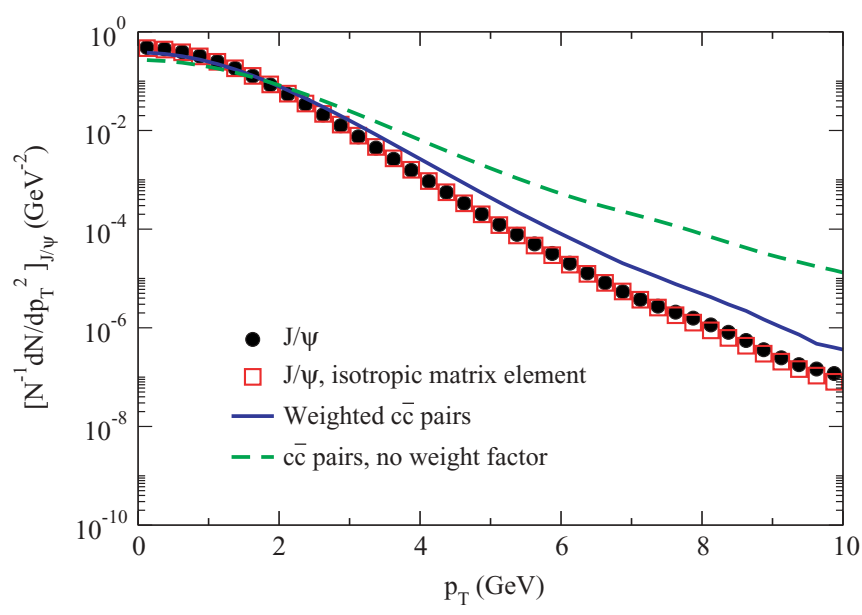

FIG. 11. (Color online) Comparison of transverse momentum spectra for $c \bar{c}$ pairs and formed $J / \psi$, all using default parameters. 


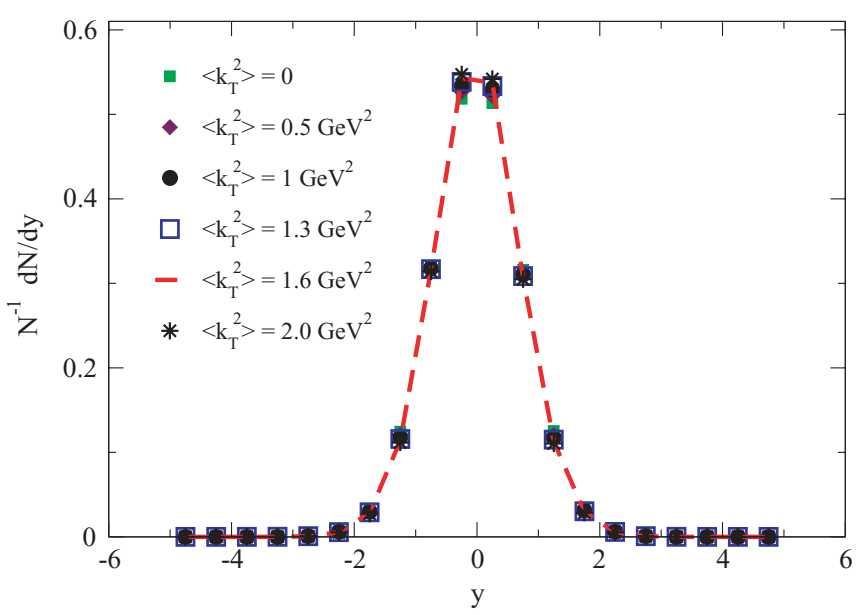

FIG. 12. (Color online) Effects of intrinsic $\left\langle k_{T}{ }^{2}\right\rangle$ on the $J / \psi$ formation rapidity spectrum.

Next we show the sensitivity of the formation results to variations in some of the default parameters. Figure 14 shows the results for the normalized transverse momentum spectra. The first three curves show variation in $d_{\max }$ (symbols). The final two curves (solid and dashed lines) show the effects of reducing the binding energy to $\epsilon_{0}=300 \mathrm{MeV}$, more than a factor of 2 below the vacuum value. In addition, the last curve has the quark mass increased to $m_{c}=1.7 \mathrm{GeV}$, which restores the $J / \psi$ mass to its vacuum value. One sees that overall these changes produce negligible variations in the $p_{T}$ spectra. Lastly, we look at extreme variations in the functional form of the cross sections to determine the degree of stability of the $J / \psi$ formation $p_{T}$ spectra. Figure 15 shows some of these effects. The effect of omitting an energy cutoff $\left(d_{\max } \rightarrow \infty\right)$ is shown by the thin solid line, which is essentially unchanged from the default parameter result (shaded circles) with $d_{\max }=3 \mathrm{GeV}$. This might be understood from the behavior of the OPE cross sections, since the entire region around the peak values is generally contained within the default parameter choices. We next consider the effect

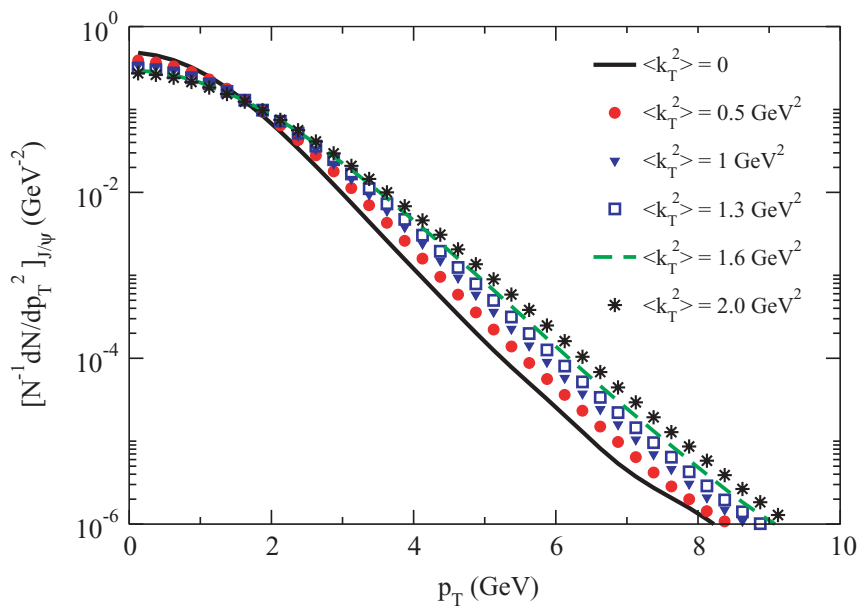

FIG. 13. (Color online) Effects of intrinsic $\left\langle k_{T}{ }^{2}\right\rangle$ on the $J / \psi$ formation transverse momentum spectrum.

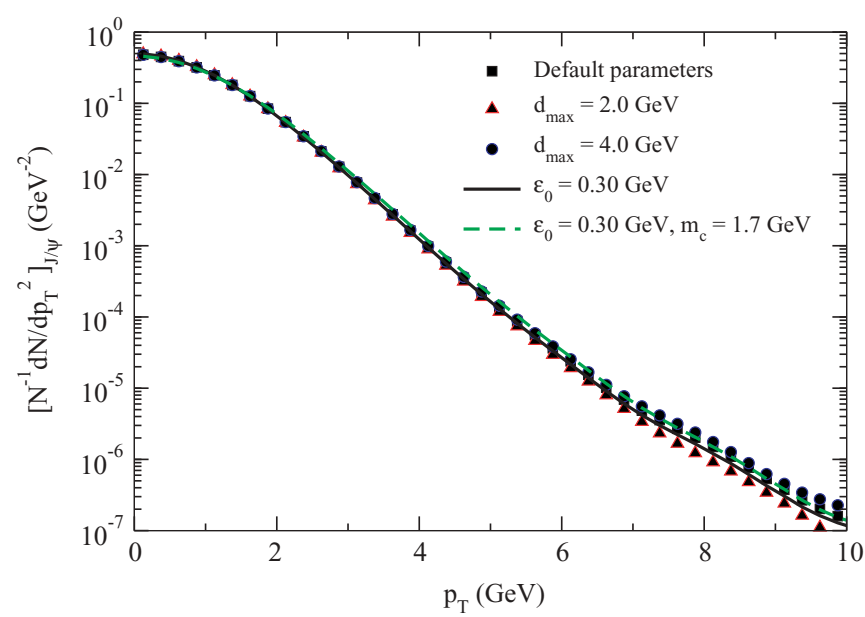

FIG. 14. (Color online) Effects of parameter variations on the $J / \psi$ formation transverse momentum spectrum.

of a cross section independent of $\sqrt{s}$, shown by the dashed line. (The calculation is virtually unchanged whether one chooses $\sigma_{F}$ or $\sigma_{D}$ to be constant). This choice of cross section produces only a small change in the $J / \psi$ transverse momentum spectrum. The most radical departure is to use constant cross sections and no energy cutoff. These results are shown by the solid line and circle, and do produce a significant change in the $p_{T}$ spectrum. We would certainly not expect, however, that the $J / \psi$ formation probability would remain independent of the $c \bar{c}$ pair relative momentum throughout the entire range populated by the $\mathrm{pQCD}$ initial production process. To the contrary, one would expect that the large invariant-mass pairs would preferentially hadronize into the higher mass states in the open charm spectrum.

Finally, we investigate the effects of in-medium dissociation on the formed $J / \psi$ spectrum. This dissociation will influence the final $p_{T}$ spectrum in two competing scenarios. In the first, one expects that the high- $p_{T} J / \psi$ initially produced will originate preferentially in central collisions, where the Cronin effect is most efficient, and thus be subject to a large

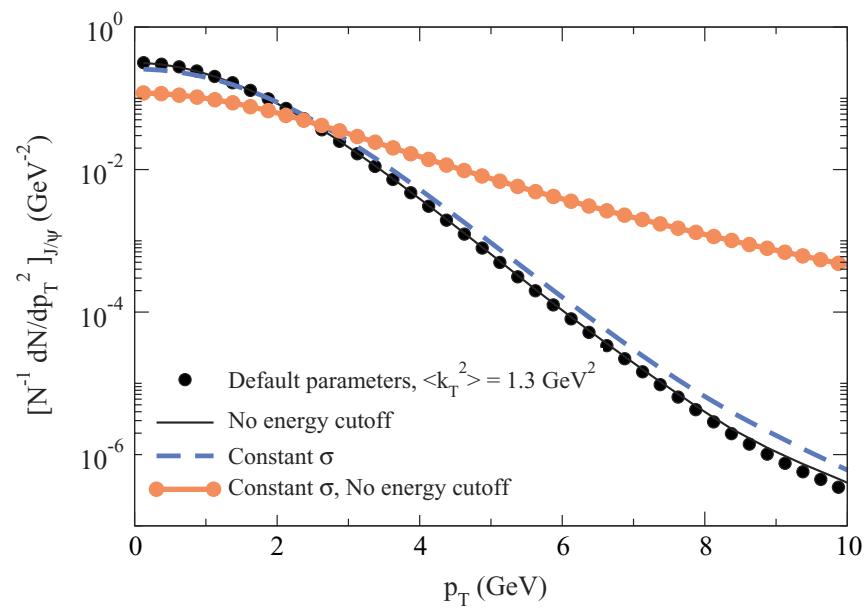

FIG. 15. (Color online) Dependence of the $J / \psi$ formation transverse momentum spectrum on the formation cross section. 


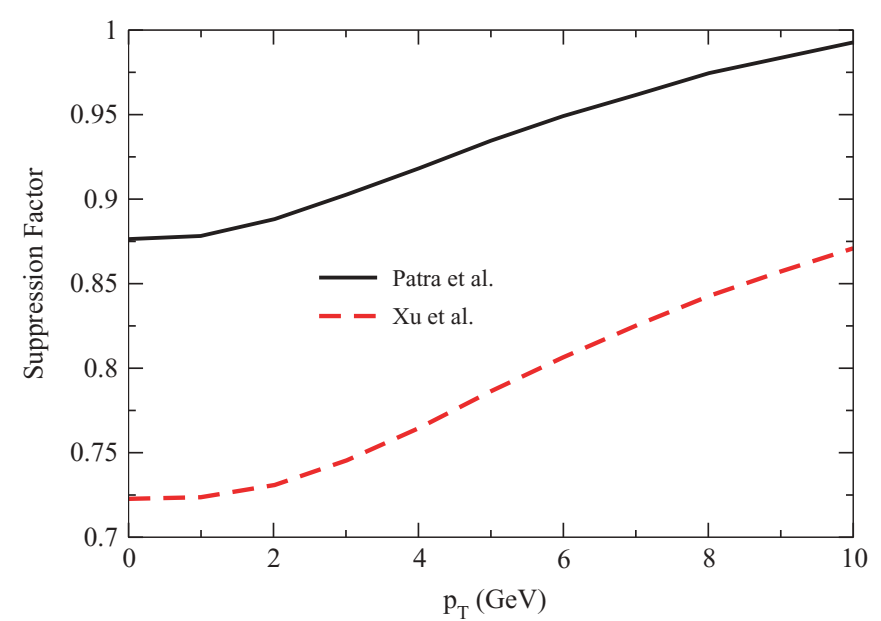

FIG. 16. (Color online) In-medium $J / \psi$ suppression factors.

suppression. However, one also expects that the $J / \psi$ with large $p_{T}$ will preferentially escape the deconfinement region. In a recent study [43] incorporating these effects for $J / \psi$ propagating in a region of deconfinement at both CERN'S Super Proton Synchroton (SPS) and RHIC energies, it was found that this second "leakage" effect dominates. We will use numerical values taken from initial studies of this effect [44] and updated versions $[45,46]$ in the form of $p_{T}$-dependent suppression factors for $J / \psi$ initially present in an equilibrating and expanding parton gas. For the application to our formation spectrum, we need a suppression factor for $J / \psi$ formed at a continuum of initial times. However, the time of formation is biased toward early times when the charm quark density is maximum. Hence, we use two typical suppression factors from Ref. [45], which have their minimum values at $p_{T}=0$ and approach unity for $p_{T} \approx 10 \mathrm{GeV}$. These are shown in Fig. 16 . The effect of these suppression factors on the formation spectra is shown in Fig. 17. One sees that the normalized formation spectra are suppressed in the low- $p_{T}$ region by $10-25 \%$. However, the normalized spectrum that includes both formation and suppression is virtually unchanged in the low- $p_{T}$ region (which dominates the normalization factor), and the entire effect of dissociation is pushed out to very large $p_{T}$. Hence, the normalized formation spectra alone provide sufficiently accurate predictions, at least in the region $0<p_{T}<6 \mathrm{GeV}$ where the majority of $J / \psi$ are found. This effect can be quantified by the small increase in $\left\langle p_{T}{ }^{2}\right\rangle$, from 3.59 to $3.69 \mathrm{GeV}^{2}$.

\section{PREDICTIONS FOR Au-Au INTERACTIONS AT RHIC}

\section{A. Charm quark distributions from pQCD}

In order to make predictions of the kinetic formation model for $J / \psi$ at RHIC, we need to fix the $\left\langle k_{T}{ }^{2}\right\rangle$ parameter. We start with $J / \psi$ production in $p p$ interactions at $200 \mathrm{GeV}$. There are PHENIX data on the rapidity and transverse momentum spectra from both run2 [30] and run3 [47], which we exhibit in Fig. 18. Since multiple $c \bar{c}$ pair production in $p p$ interactions is negligible, we consider only the diagonal $c \bar{c}$ pairs, which are

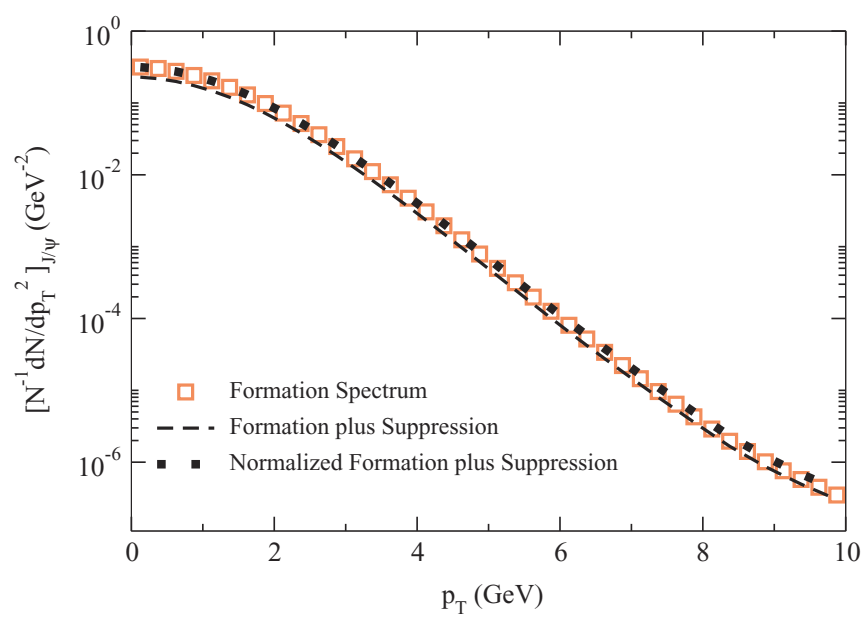

FIG. 17. (Color online) In-medium $J / \psi$ normalized formation plus suppression spectrum compared with the normalized formationonly spectrum.

shown by the solid curve: They are seen to describe the data reasonably well. (Recall from Fig. 7 that the $c \bar{c}$ pair rapidity spectra are essentially independent of $\left\langle k_{T}{ }^{2}\right\rangle$.) One can interpret this agreement as being consistent with any mechanism in which $J / \psi$ is produced via hadronization of initially produced $c \bar{c}$ pairs. The dotted curve shows the pairs modified by the in-medium formation probability, proportional to $\sigma_{F}$. This process, of course, cannot occur in a $p p$ interaction, and it is gratifying to see that it is incompatible with the data.

The transverse momentum spectrum is shown in Fig. 19. The PHENIX dimuon data are presented for both positive and negative rapidities, which have smaller uncertainties than the central dielectron data. The solid triangles are the pQCD $c \bar{c}$ diagonal pairs, which, of course, must be augmented by some $\left\langle k_{T}{ }^{2}\right\rangle$ to include initial state and confinement effects. The solid line and adjacent circles and diamonds with $\left\langle k_{T}{ }^{2}\right\rangle=0.5 \pm 0.1 \mathrm{GeV}^{2}$ provide an acceptable fit to the data (fluctuations at large $p_{T}$ are statistics limited by the number of generated diagonal pQCD events).

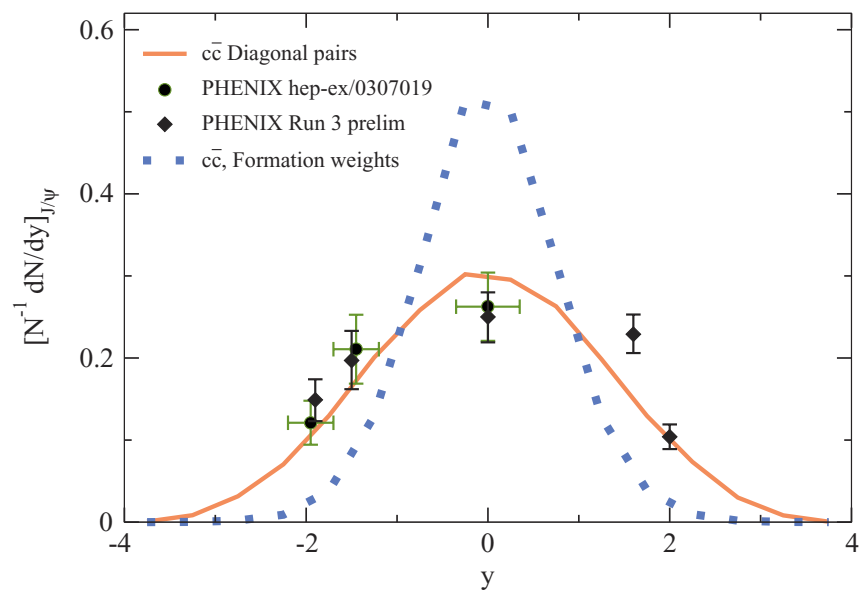

FIG. 18. (Color online) Rapidity spectra of $J / \psi$ production in $p p$ interactions at $200 \mathrm{GeV}$. 


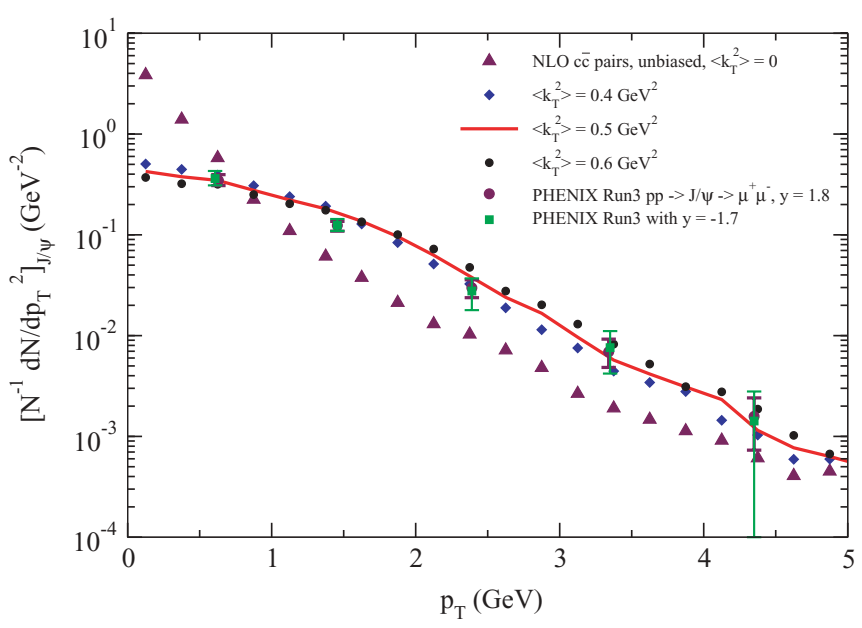

FIG. 19. (Color online) Transverse momentum spectra of $J / \psi$ production in $p p$ interactions at $200 \mathrm{GeV}$.

There are also preliminary data on $J / \psi$ production in $d$-Au interactions [47]. The width of the $p_{T}$ spectra is found to be larger than that measured in $p p$ interactions:

$\left\langle p_{T}{ }^{2}\right\rangle_{d-\mathrm{Au}}-\left\langle p_{T}{ }^{2}\right\rangle_{p p}=\left\{\begin{array}{l}1.77 \pm 0.35 \mathrm{GeV}^{2}(y=-1.7), \\ 1.29 \pm 0.35 \mathrm{GeV}^{2}(y=+1.8) .\end{array}\right.$

This well-known broadening of $p_{T}$ distributions for particles produced on nuclear targets fits a natural pattern that emerges from initial state elastic scattering of a projectile in the nuclear target [48]. For $p A$ (or $d A$ ) interactions, the increase can be expressed as a change in $\left\langle p_{T}{ }^{2}\right\rangle$, as

$$
\left\langle p_{T}{ }^{2}\right\rangle_{p A}-\left\langle p_{T}{ }^{2}\right\rangle_{p p}=\lambda^{2}\left[\bar{n}_{A}-1\right],
$$

where $\bar{n}_{A}$ is the impact-averaged number of inelastic interactions of the projectile in nucleus $A$, and $\lambda^{2}$ is the square of the transverse momentum transfer per collision. For a nucleus-nucleus collision, the corresponding relation is

$$
\left\langle p_{T}{ }^{2}\right\rangle_{A B}-\left\langle p_{T}{ }^{2}\right\rangle_{p p}=\lambda^{2}\left[\bar{n}_{A}+\bar{n}_{B}-2\right] .
$$

We use the measured $J / \psi$ broadening in $d$-Au to determine the appropriate $\left\langle k_{T}{ }^{2}\right\rangle$ value for the $p_{T}$ distribution of initially produced diagonal $c \bar{c}$ pairs through Eq. (6) or (8), with the result $\left\langle k_{T}{ }^{2}\right\rangle_{\mathrm{d}-A u}-\left\langle k_{T}{ }^{2}\right\rangle_{p p}=0.4 \pm 0.1$. (Since the measured values for $d$-Au interactions differ between positive and negative rapidity, we use their average to partially compensate for the existence of final state effects.) Finally, using $\left\langle k_{T}{ }^{2}\right\rangle_{p p}$ and $\left\langle k_{T}{ }^{2}\right\rangle_{d \text {-Au }}$ extracted from data, in combination with Eq. (17), leads to $\left\langle k_{T}{ }^{2}\right\rangle_{\mathrm{Au}-\mathrm{Au}}=1.3 \pm 0.3 \mathrm{GeV}^{2}$. We use this value to determine the $p_{T}$ distribution of initially produced charm quarks in $\mathrm{Au}-\mathrm{Au}$ interactions. It is interesting to also look at the equivalent parameters which follow from Eqs. (15) and (16). One finds $\bar{n}_{A}=5.4$ for minimum bias $d$-Au interactions at RHIC energy (using $\sigma_{p p}=42 \mathrm{mb}$ ), which leads to $\lambda^{2}=0.35 \pm 0.14 \mathrm{GeV}^{2}$. We note that the relatively large uncertainty comes entirely from the difference in $p_{T}$ broadening in $d$-Au between positive and negative rapidity. It is interesting to see the energy dependence by comparing this value with that extracted from $J / \psi p A$ data at fixed-target energy [48], $\lambda^{2}=0.12 \pm 0.02 \mathrm{GeV}^{2}$.

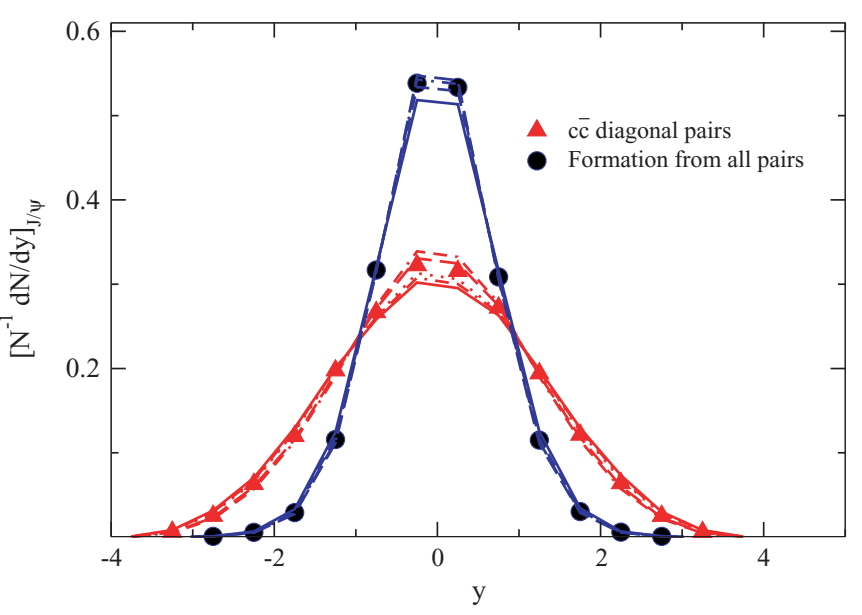

FIG. 20. (Color online) Predicted rapidity spectra of $J / \psi$ in Au$\mathrm{Au}$ interactions at $200 \mathrm{GeV}$. Triangles are for initial production via diagonal $c \bar{c}$ pairs. Circles are for in-medium formation via all pairs. Sensitivity of the formation spectrum to variation of $\left\langle k_{T}{ }^{2}\right\rangle$ within the range $\left\langle k_{T}{ }^{2}\right\rangle=1.3 \pm 0.3 \mathrm{GeV}^{2}$ is indicated by the spread in the solid lines. The corresponding spread in the lines for diagonal pairs covers the range $0<\left\langle k_{T}{ }^{2}\right\rangle<2 \mathrm{GeV}^{2}$.

Shown in Fig. 20 are the predicted rapidity spectra of $J / \psi$ in Au-Au interactions at $200 \mathrm{GeV}$. The solid triangles give the results for initially produced diagonal $c \bar{c}$ pairs with $\left\langle k_{T}{ }^{2}\right\rangle=$ $1.3 \mathrm{GeV}^{2}$, and the various lines indicate the small sensitivity to variations of $\left\langle k_{T}^{2}\right\rangle_{\mathrm{Au}-\mathrm{Au}}$ within uncertainties. This distribution is also virtually the same as those using $\left\langle k_{T}{ }^{2}\right\rangle_{p p}$, which fit the $p p$ data as shown in Fig. 18. The solid circles and associated lines show the results of a formation calculation using all $c \bar{c}$ pairs that can recombine in the medium. These spectra are substantially narrower and provide a prediction which signals the existence of the formation mechanism. Since the formation process is largest for central collisions, where dissociation of the initial production yield should be most efficient, one would expect that the total $J / \psi$ rapidity spectrum should change from narrow for the most central collisions to wide for the most peripheral collisions.

Figure 21 shows the corresponding transverse momentum spectra. The solid triangles again use initially produced diagonal $c \bar{c}$ pairs with $\left\langle k_{T}{ }^{2}\right\rangle_{\mathrm{Au}-\mathrm{Au}}=1.3 \mathrm{GeV}^{2}$. Note that this distribution is wider than those using $\left\langle k_{T}{ }^{2}\right\rangle_{p p}$, which fit the $p p$ data shown in Fig. 19. The solid circles and associated lines (which include the uncertainty in $\left\langle k_{T}{ }^{2}\right\rangle_{\mathrm{Au}-\mathrm{Au}}$ ) show the results of a formation calculation using all $c \bar{c}$ pairs that can recombine in the medium. These spectra are substantially narrower and provide another prediction which signals the existence of the formation mechanism. The same consideration of centrality dependence as presented for the rapidity spectra also applies to these transverse momentum spectra.

\section{B. Charm quark distributions from thermal plus flow}

The calculation of $J / \psi$ follows from the same cross section used for the pQCD charm quark distributions. We use generated $c \bar{c}$ events as calculated in Sec. III C, where it was demonstrated that the charm quark transverse momentum 


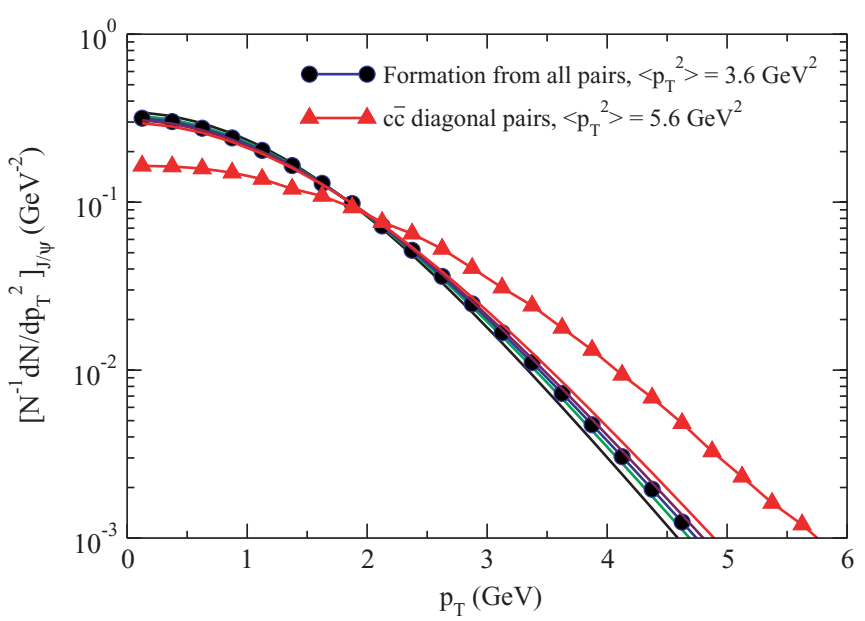

FIG. 21. (Color online) Same as Fig. 20, but for transverse momentum spectra of $J / \psi$ in Au-Au interactions at $200 \mathrm{GeV}$.

distributions which follow from thermal plus flow parameters $T=128 \mathrm{MeV}, y_{T \max }=0.65$ and $T=170 \mathrm{MeV}, y_{T \max }=$ 0.50 are essentially identical.

We show in Fig. 22 the transverse momentum spectra evolution which follows from charm quarks with $T=170 \mathrm{MeV}$, $y_{T \max }=0.50$. One sees a downward progression in widths, starting with $\left\langle p_{T}{ }^{2}\right\rangle=2.5 \mathrm{GeV}^{2}$ for $c \bar{c}$ pairs, to $2.0 \mathrm{GeV}^{2}$ for weighted $c \bar{c}$ pairs and $1.7 \mathrm{GeV}^{2}$ for $J / \psi$ formation. It is important to note that the concept of diagonal pairs no longer exists for this set of charm quarks, since their formation identity has been entirely erased during the thermalization process. As might have been expected with a thermal distribution, these widths are all smaller than any of those which follow from pQCD charm quark distributions. This is shown in Fig. 23. The filled diamonds and triangles are the $J / \psi$ formation and diagonal $c \bar{c}$ pair distributions which follow from the pQCD quark distributions as calculated in Sec. V A. The solid squares and dashed line are for the two equivalent thermal plus flow charm quark distributions, and

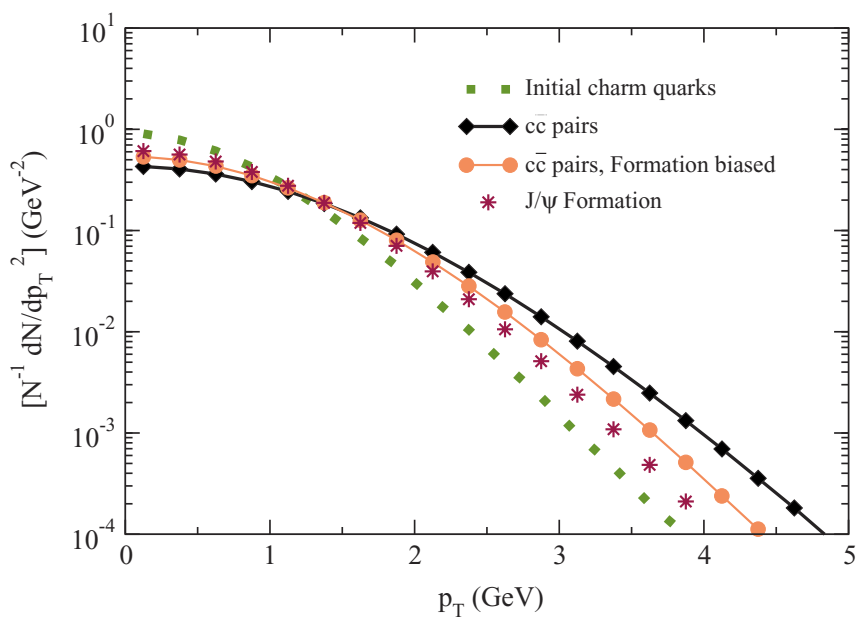

FIG. 22. (Color online) Transverse momentum spectra comparison for $J / \psi$ and $c \bar{c}$ pairs for a thermal plus flow initial quark distribution.

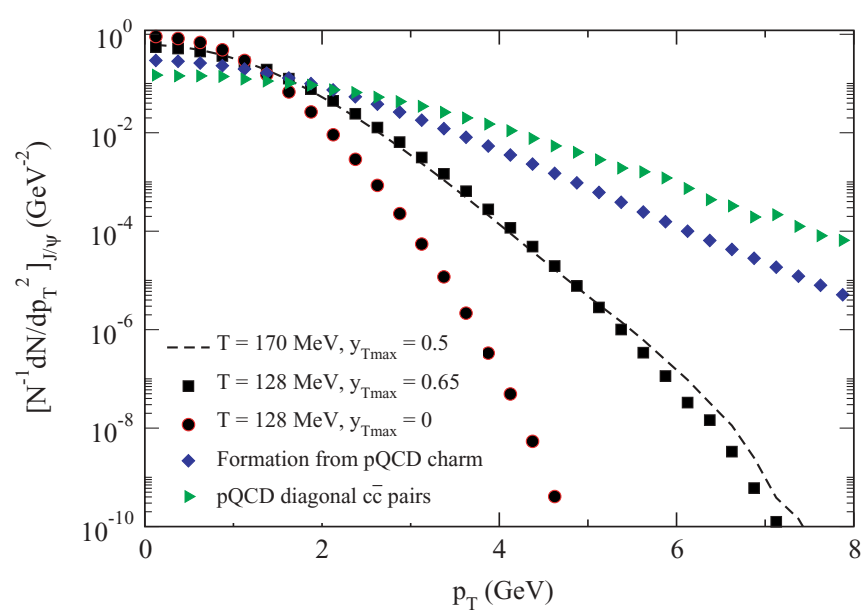

FIG. 23. (Color online) $J / \psi$ formation transverse momentum spectra dependence on initial charm quark distributions.

for completeness a formation spectra without charm quark transverse flow is shown by the solid circles. It is clear that in-medium formation of $J / \psi$ starting from either the pQCD or thermal plus flow charm quark distributions predict transverse momentum widths (characterized by $\left\langle p_{T}^{2}\right\rangle$ ) which are markedly smaller than those for initially produced $J / \psi$. The difference between the two formation possibilities would be reflected by the significant difference between the shapes of the $p_{T}$ distributions.

In Fig. 24, we compare these results with alternate model calculations in the literature. The filled diamonds and squares are the $J / \psi$ transverse momentum spectra from our in-medium formation calculations using the two extreme assumptions about the charm quark momentum distributions, pQCD (diamonds) and thermal plus flow (squares). We compare this with a calculation that uses a coalescence model to produce $J / \psi$ from charm quark pairs [49]. The solid line is the result using charm spectra generated by Pythia (compare with our pQCD formation), and the dashed line uses thermal plus flow charm spectra in the coalescence model

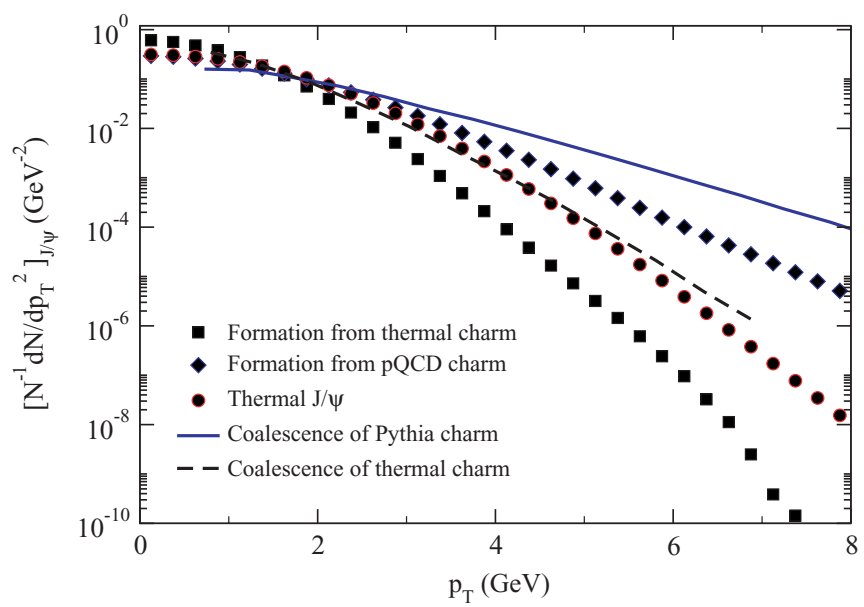

FIG. 24. (Color online) $J / \psi$ formation transverse momentum spectra comparison with alternative models. 
(compare also with our formation using thermal plus flow). One sees that the systematics of the model spectra are the same, but the coalescence process yields somewhat broader spectra than our direct in-medium formation. Also shown by the filled circles is the $J / \psi$ spectrum which follows from a statistical model of hadronization. Here the $J / \psi$ would inherit a thermal plus flow kinetic distribution [50]. This distribution is broader than the underlying charm quark thermal plus flow case, but narrower than the pQCD formation and coalescence processes. The width of the $J / \psi$ transverse momentum spectra at RHIC has also been predicted in [43]. The value quoted for the most central collisions is $\left\langle p_{T}{ }^{2}\right\rangle=3.3 \mathrm{GeV}^{2}$ for the most central collisions. For comparison, our pQCD formation process predicts $3.6 \mathrm{GeV}^{2}$ in a region where it is the dominant process.

\section{SUMMARY}

We have shown that in-medium formation of heavy quarkonium states utilizing charm and anticharm quarks coming from independent hard scatterings results in normalized momentum spectra which retain a memory of the underlying quark distributions. In the case that the quark distributions follow from initial production in pQCD processes, both the rapidity and transverse momentum spectra of the formed heavy quarkonium are narrower than those expected from diagonal pairs in the absence of a color-deconfined medium. Explicit calculations for $J / \psi$ formation in $\mathrm{Au}-\mathrm{Au}$ collisions at $200 \mathrm{GeV}$ were performed, using initial data in $p p$ and $d$-Au interactions to fix some parameters. A striking feature is the nonmonotonic behavior of the transverse momentum spectrum widths $\left\langle p_{T}{ }^{2}\right\rangle$ in the progression $p p$ to $p A$ to $A A$. In the absence of in-medium formation, one would expect $\left\langle p_{T}{ }^{2}\right\rangle$ to increase monatonically with the colliding system size, because of the initial state confinement and nuclear broadening effects. Our calculations for in-medium formation in $A A$ collisions predict $\left\langle p_{T}{ }^{2}\right\rangle$ substantially smaller than expected if the $J / \psi$ were due to initial production (see Fig. 21). In fact, the predicted $\left\langle p_{T}{ }^{2}\right\rangle_{A A}=3.59 \mathrm{GeV}^{2}$ is even smaller than the measured value in $d$-Au interactions, $\left\langle p_{T}^{2}\right\rangle_{d-\mathrm{Au}}=4.25 \mathrm{GeV}^{2}$.

This effect is maximum for central $A A$ collisions and reverts to the "normal" behavior for peripheral collisions. Uncorrelated pairs, composed of one quark and one antiquark which do not originate from the same initial interaction, have an invariant-mass spectrum which becomes harder for larger $p_{T}$ of the pair. Given that the charmonium formation dynamics favors lighter invariant masses, we expect that the contribution of uncorrelated pairs will be more dominant at smaller $p_{T}$, leaving a signature in the overall $p_{T}$ slope. The softening of the $p_{T}$ spectrum for uncorrelated pairs, in other words, is the result of an increased contribution to charmonium production at lower $p_{T}$.

We also considered the formation process using thermal charm quarks which flow with the expanding medium. The resulting spectra are substantially narrower and retain a form which reflects that of the underlying heavy quarks. Overall, our normalized spectra appear to be quite robust with respect to variations of the model parameters, independent of the absolute magnitude of in-medium formation.

\section{ACKNOWLEDGMENT}

This research was partially supported by the U.S. Department of Energy under Grant No. DE-FG02-04ER41318.
[1] T. Matsui and H. Satz, Phys. Lett. B178, 416 (1986).

[2] P. Braun-Munzinger and J. Stachel, Phys. Lett. B490, 196 (2000).

[3] R. L. Thews, M. Schroedter, and J. Rafelski, Phys. Rev. C 63, 054905 (2001).

[4] R. L. Thews, Nucl. Phys. A702, 341 (2002).

[5] R. Gavai, D. Kharzeev, H. Satz, G. A. Schuler, K. Sridhar, and R. Vogt, Int. J. Mod. Phys. A 10, 3043 (1995).

[6] S. S. Adler et al. (PHENIX Collaboration), Phys. Rev. Lett. 94, 082301 (2005).

[7] J. Adams et al. (STAR Collaboration), Phys. Rev. Lett. 94, 062301 (2005).

[8] R. Vogt, J. Phys. G 31, S773 (2005).

[9] A. Andronic, P. Braun-Munzinger, K. Redlich, and J. Stachel, Phys. Lett. B571, 36 (2003).

[10] A. P. Kostyuk, M. I. Gorenstein, H. Stocker, and W. Greiner, Phys. Rev. C 68, 041902(R) (2003).

[11] M. Schroedter, R. L. Thews, and J. Rafelski, Phys. Rev. C 62, 024905 (2000).

[12] R. L. Thews, M. Schroedter, and J. Rafelski, J. Phys. G 27, 715 (2001).

[13] R. L. Thews and J. Rafelski, Nucl. Phys. A698, 575 (2002).
[14] S. Datta, F. Karsch, P. Petreczky, and I. Wetzorke, Phys. Rev. D 69, 094507 (2004).

[15] M. Asakawa and T. Hatsuda, Phys. Rev. Lett. 92, 012001 (2004).

[16] L. Grandchamp and R. Rapp, Phys. Lett. B523, 60 (2001).

[17] L. Grandchamp and R. Rapp, Nucl. Phys. A709, 415 (2002).

[18] L. Grandchamp, R. Rapp, and G. E. Brown, Phys. Rev. Lett. 92, 212301 (2004).

[19] R. L. Thews, J. Phys. G 30, S369 (2004).

[20] D. Kharzeev and H. Satz, Phys. Lett. B334, 155 (1994).

[21] M. E. Peskin, Nucl. Phys. B156, 365 (1979).

[22] G. Bhanot and M. E. Peskin, Nucl. Phys. B156, 391 (1979).

[23] D. Kharzeev, arXiv:nucl-th/9601029.

[24] X. M. Xu, C. Y. Wong, and T. Barnes, Phys. Rev. C 67, 014907 (2003).

[25] K. Martins, D. Blaschke, and E. Quack, Phys. Rev. C 51, 2723 (1995).

[26] F. S. Navarra, M. Nielsen, R. S. Marques de Carvalho, and G. Krein, Phys. Lett. B529, 87 (2002).

[27] L. Maiani, F. Piccinini, A. D. Polosa, and V. Riquer, Nucl. Phys. A741, 273 (2004).

[28] B. Zhang, C. M. Ko, B. A. Li, Z. W. Lin, and S. Pal, Phys. Rev. C 65, 054909 (2002). 
[29] S. S. Adler et al. (PHENIX Collaboration), Phys. Rev. C 69, 014901 (2004).

[30] S. S. Adler et al. (PHENIX Collaboration), Phys. Rev. Lett. 92, 051802 (2004).

[31] M. L. Mangano, P. Nason, and G. Ridolfi, Nucl. Phys. B373, 295 (1992).

[32] K. J. Eskola, V. J. Kolhinen, and P. V. Ruuskanen, Nucl. Phys. B535, 351 (1998).

[33] M. Cacciari, P. Nason, and R. Vogt, Phys. Rev. Lett. 95, 122001 (2005).

[34] M. L. Mangano, P. Nason, and G. Ridolfi, Nucl. Phys. B405, 507 (1993); B431, 453 (1994); Adv. Ser. Direct. High Energy Phys. 15, 609 (1998).

[35] Y. L. Dokshitzer and D. E. Kharzeev, Phys. Lett. B519, 199 (2001).

[36] K. Adcox et al. (PHENIX Collaboration), Phys. Rev. Lett. 88, 192303 (2002).

[37] S. Batsouli, S. Kelly, M. Gyulassy, and J. L. Nagle, Phys. Lett. B557, 26 (2003).
[38] H. van Hees and R. Rapp, Phys. Rev. C 71, 034907 (2005).

[39] E. Schnedermann, J. Sollfrank, and U. Heinz, Phys. Rev. C 48, 2462 (1993).

[40] K. A. Bugaev, M. Gazdzicki, and M. I. Gorenstein, Phys. Rev. C 68, 017901 (2003).

[41] V. Greco, C. M. Ko, and P. Levai, Phys. Rev. C 68, 034904 (2003).

[42] C. Y. Wong, J. Phys. G 28, 2349 (2002).

[43] X. 1. Zhu, P. F. Zhuang, and N. Xu, Phys. Lett. B607, 107 (2005).

[44] X. M. Xu, D. Kharzeev, H. Satz, and X. N. Wang, Phys. Rev. C 53, 3051 (1996).

[45] B. K. Patra and V. J. Menon, Eur. Phys. J. C 37, 115 (2004).

[46] B. K. Patra and V. J. Menon, Eur. Phys. J. C 44, 567 (2005).

[47] R. G. de Cassagnac (PHENIX Collaboration), J. Phys. G 30, S1341 (2004).

[48] S. Gavin and M. Gyulassy, Phys. Lett. B214, 241 (1988).

[49] V. Greco, C. M. Ko, and R. Rapp, Phys. Lett. B595, 202 (2004).

[50] K. A. Bugaev, M. Gazdzicki, and M. I. Gorenstein, Phys. Lett. B544, 127 (2002). 